\section{$\underset{\substack{\text { hommes } \\ \text { \& migrations }}}{ }$}

\section{Hommes \& migrations}

Revue française de référence sur les dynamiques

migratoires

\section{$1309 \mid 2015$}

Le $3 e$ âge des migrants

\title{
"Family values"
}

La dépendance aux transferts de fonds et le dilemme du retour au pays à un âge avancé

\section{Alistair Hunter}

\section{OpenEdition}

\section{Journals}

Édition électronique

URL : http://journals.openedition.org/hommesmigrations/3092

DOI : 10.4000/hommesmigrations.3092

ISSN : 2262-3353

\section{Éditeur}

Musée national de l'histoire de l'immigration

\section{Édition imprimée}

Date de publication : 1 janvier 2015

Pagination : 117-125

ISBN : 978-2-919040-30-8

ISSN : $1142-852 X$

\section{Référence électronique}

Alistair Hunter, " "Family values"», Hommes \& migrations [En ligne], 1309 | 2015, mis en ligne le 01

janvier 2017, consulté le 21 avril 2019. URL : http://journals.openedition.org/hommesmigrations/3092 ; DOI : 10.4000/hommesmigrations.3092 


\title{
"FAMILY VALUES" \\ LA DÉPENDANCE AUX TRANSFERTS DE FONDS ET LE DILEMME DU RETOUR AU PAYS À UN ÂGE AVANCÉ
}

par ALISTAIR HUNTER, chercheur post-doctorant à l'université d'Edimbourg'.

\author{
D'une manière assez inattendue, la majorité des travailleurs \\ immigrés originaires d'Afrique du Nord et d'Afrique de I'Ouest \\ logés dans les foyers ne voient pas dans la retraite l'occasion \\ d'un retour définitif dans leur pays d'origine. Une approche \\ croisant le genre, l'âge, la génération et l'origine ethnique permet \\ de comprendre la façon dont ils vieillissent en foyer. \\ Qu'ils affirment leur rôle de chef de famille à distance ou, \\ au contraire, que l'évolution des structures familiales \\ en leur absence vienne à diminuer leur influence, ces hommes \\ sont contraints d'habiter un entre-deux.
}

Au tournant du XXI e siècle, la France comptait environ 80000 travailleurs migrants de sexe masculin répartis dans quelque 700 foyers $^{2}$ à travers tout le pays. $63 \%$ d'entre eux étaient originaires d'Afrique du Nord et $28 \%$ d'Afrique de l'Ouest. Si ces foyers continuent d'opérer, ce n'est pas, mis à part quelques exceptions, parce que les jeunes migrants ont remplacé leurs aînés. Au contraire, la grande majorité des résidents actuels de ces foyers ont "vieilli dans les murs"." En 2005, lâge moyen des résidents était 55 ans ; en 2013, un résident sur trois était âgé de plus de 65 ans, avec au total environ 35000 immigrés vieillissants ${ }^{4}$.

Une fois à la retraite, et à la surprise générale, tant des responsables politiques français et du gouvernement du pays d'origine que des familles de ces hommes, de nombreux résidents ne sont pas allés rejoindre définitivement leur femme et leurs enfants restés au pays. Au lieu de rentrer chez eux, la majorité des résidents retraités circulent 
régulièrement entre le foyer en France et leur pays d'origine, séjournant plus ou moins longtemps aux deux endroits. Selon des enquêtes, entre 80 et $95 \%$ des résidents font des allers-retours entre la France et leur pays d'origine à l'âge de la retraite ${ }^{5}$.

Outre le pouvoir supposé des liens affectifs, les résidents vieillissants des foyers de travailleurs migrants ont également déjoué les attentes des théoriciens de la migration, puisque ces hommes sont restés insensibles aux incitations financières d'un retour définitif au pays,

Au lieu de rentrer chez eux,

la majorité des résidents

retraités circulent

régulièrement entre le foyer en France et leur pays d'origine,

séjournant plus ou moins longtemps aux deux endroits. où leur retraite française, payée en euros et entièrement transférable, leur offrirait un pouvoir d'achat bien plus conséquent ${ }^{6}$. Cette absence de retour définitif à l'âge de la retraite est surprenante non seulement pour

les économistes néoclassiques, mais aussi pour les chercheurs étudiant la question sous l'angle du "mythe du retour". Pour ces derniers, le non-retour dans le pays d'origine s'explique par le regroupement familial et la scolarisation des enfants dans le pays d'accueil'. Or cette explication n'est pas valable dans notre cas précis puisque les résidents vieillissants des foyers n'ont justement pas fait ce regroupement. Bien que les raisons qui les poussent à préférer les déplacements au retour définitif soient multiples (raisons de santé ou contraintes juridicoadministratives d'affiliation à la Sécurité sociale française ${ }^{8}$ ), nous nous limiterons dans cet article à analyser les changements survenus dans les familles transnationales, notamment du point de vue du genre et des relations intergénérationnelles. L'analyse intersectionnelle se prête particulièrement à cette mission. Au cœur du contexte d'intersectionnalité réside l'idée que les phénomènes de discrimination et d'inégalité liés notamment au genre et à l'origine ethnique ne peuvent pas être subsumés ou "isolés dans des catégories pures et à part ${ }^{9 "}$. Si la littérature intersectionnelle est d'abord apparue dans les études féministes des discriminations raciales ${ }^{10}$, d'autres chercheurs ont tenté ces dernières années d'étendre le champ du concept et à y inclure lâge, la classe, le handicap et la sexualité comme autant de critères de différenciation et d'inégalité ${ }^{11}$. En étudiant les motivations de cette (im)mobilité à l'âge de la retraite dans une perspective où s'entremêlent différentes dimensions de la stratification sociale - à savoir : le genre, lâge, la génération et l'origine ethnique -, la complexité des relations qu'entretiennent les personnes interrogées avec leur famille transnationale émerge progressivement.

Cet article tentera de montrer comment le modèle social et économique prédominant de breadwinner (soutien de famille), un modèle genré et propre à une génération en particulier, perdure à la retraite, forçant certains résidents vieillissants à le perpétuer, et devenant, par conséquent, un frein au retour définitif. Toutefois, la prise en compte de l'origine ethnique permet de nuancer ce constat en faisant apparaître des différences entre les résidents nord-africains et ouest-africains qui mériteraient d'être examinées en profondeur.

L'analyse que nous présentons ici s'appuie sur des données recueillies dans le cadre d'un modèle de recherche ethnographique. Ce dernier comprend une observation participante faite au sein de foyers de travailleurs migrants en tant que résident, puis au sein des familles en tant qu'invité accompagnant certains des résidents lors de leurs séjours familiaux au Maroc et au Sénégal.

5. Françoise Bitatsi Trachet, Mohammed El Moubaraki, "Prise en charge du vieillissement de la population en foyers de travailleurs migrants et résidences sociales", in Migrations Santé, n 127-8, 2006, pp. 97-114 ; Sonacotra, "Problématiques liées à la pratique de l'aller-retour", Paris, Direction de la clientèle, département Marketing et commercial, études et développement, Sonacotra, 2006. 6. Martin Klinthäll, "Retirement return migration from Sweden”, in International Migration, vol 44, $\mathrm{n}^{\circ}$ 2, 2006, pp. 153-180. 7. Muhammad Anwar, The Myth of Return. Pakistanis in Britain, London, Heinemann, 1979.

8. Pour de plus amples informations, voir Alistair Hunter, "Theory and practice of return migration at retirement: the case of migrant worker hostel residents in France", in Population, Space and Place, vol. 17, n² 2, 2011, pp. 179-192.

9. Avtar Brah, Ann Phoenix, "Ain't I a woman? Revisiting intersectionality", in Journal of International Women's Studies, vol. 5 , $n^{\circ}$ 3, 2004, p. 76. 10. Bell Hooks, Ain't I a Woman, Boston, South End Press, 1981. 11. Harriet Bradley, Fractured identities. Changing patterns of inequality, Cambridge, Polity, 1996 ; Avtar Brah, Ann Phoenix, op. cit. ; Nira Yuval-Davis, "Intersectionality and feminist politics", in European Journal of Women's Studies, vol. 13, n³ 3, 2006, pp. 193 -209. 
Cette méthode intègre également une dimension relative au parcours de vie en faisant appel à des techniques d'entretien biographique. Au total, quelque 25 résidents ont accepté de nous accorder un entretien biographique nous permettant de faire une analyse exploratoire des relations familiales des migrants à la retraite.

\section{Contextualisation des flux migratoires et des régions d'origine}

Comme nous l'avons mentionné, un peu moins de deux tiers des résidents migrants sont issus d'Afrique du Nord, et un peu moins d'un tiers viennent d'Afrique de l'Ouest. Malgré certains points communs, notamment la prépondérance de jeunes hommes, qui caractérisait les deux flux migratoires jusqu'aux années $1980^{12}$, il existe des différences entre les migrations d'Afrique du Nord et d'Afrique de l'Ouest, la plus importante concernant les positions envers les communautés d'origine. Ce qui est remarquable dans l'expérience ouest-africaine, du moins dans la région émettrice soninké de la vallée du fleuve Sénégal, c'est la place centrale que ne cesse d'occuper le village ou la ville d'origine pour les communautés émigrantes en France $^{13}$. Cela s'explique en partie par les traditions d'organisation sociale de ces communautés.

Bien avant la mise en place de l'émigration transnationale à grande échelle, il existait des mécanismes de solidarité dans les villages soninkés, notamment des activités visant à réduire la destruction des cultures par les sauterelles, le bétail ou les oiseaux. Les semailles et les récoltes étaient des travaux collectifs. Cette solidarité a persisté face à l'émergence des nouveaux défis de la vie, tels que les périodes de sécheresse ou la marginalisation économique de la part du gouvernement central ${ }^{14}$. Par contraste, les émigrés nord-africains se concentrent sur une échelle plus étroite, celle de la cellule familiale. Selon Abdelmalek Sayad, à mesure que les communautés ont commencé à être concernées par l'émigration, en particulier par le passage à une économie monétisée, les obligations traditionnelles envers la communauté au sens large et les terres possédées en commun ont été remplacées par des allégeances plus étroites, circonscrites à la famille proche $e^{15}$.

\section{La dépendance aux transferts de fonds après la retraite}

La préférence des résidents retraités pour une migration pendulaire plutôt que pour un retour définitif est déroutante du point de vue de l'économie néoclassique ${ }^{16}$. Il ressort des entretiens que cela n'a aucun sens pour les personnes interrogées de parler de coût et d'avantages personnels : ces perceptions de richesse individuelle sont tempérées par le rôle Le devoir de subvenir aux besoins d'un nombre de breadwinner que les résidents important de personnes des foyers sont censés tenir et par à charge après la retraite l'ampleur des responsabilités qu'ils pèse lourdement. ont envers leurs proches dans leur pays d'origine. Ces responsabilités pèsent plus lourd sur les personnes interrogées une fois à la retraite: "On vit dans cette solitude, mais là-bas, il y a plus de responsabilités. Ici [en France] on s'occupe de soi-même, les soins, etc., mais, là-bas, c'est le père, c'est le chef de famille qui doit s'occuper de tout le monde, de tout type de problèmes." (Waly, 75 ans, Mali ${ }^{17}$ ).

Le devoir de subvenir aux besoins d'un nombre important de personnes à charge après la retraite pèse lourdement. Les personnes interrogées ont souvent mentionné les nombreux proches restant à leur charge financière après la retraite. Ainsi, Issa (Tambacounda, Sénégal), 70 ans, a toujours sept enfants vivant à la maison, dont certains sont encore scolarisés ; Hadyatou (Matam, Sénégal), 
72 ans, a cinq enfants qui vont à l'école, dont trois à l'école primaire. Par conséquent, le départ en retraite ne s'accompagne pas toujours d'un passage de relais de la responsabilité financière aux jeunes générations, à l'inverse de ce qui est prévu dans le paradigme appelé "la nouvelle économie de la migration de travail" (NEMT) ${ }^{18}$. La famille continue de dépendre des transferts de fonds des résidents des foyers, même une fois terminée leur vie active. La baisse de leurs revenus à la retraite et le manque à gagner que cela représente pour le budget de leur famille forcent de nombreux résidents à réclamer l'allocation de solidarité aux personnes âgées (communément désignée sous le terme de "minimum vieillesse"). Cette allocation sociale, sous conditions de ressources, permet de compléter la pension de retraite et de bénéficier d'un revenu minimum d'existence fixé à 708,95 euros par mois en 2010 (année à laquelle ces travaux de recherche ont été menés).

Si cette prestation permet à leur famille de s'en sortir financièrement, elle oblige en contrepartie les résidents retraités à respecter les critères de résidence, à savoir l'obligation de résider plus de six mois en France par année calendaire, prolongeant ainsi la séparation géographique d'avec les leurs ${ }^{19}$. Ces questions de dépendance aux transferts de fonds "piègent" pour la majeure partie de l'année en France certains résidents qui ont besoin du minimum vieillesse pour compléter leur retraite. Saleem, notamment, est revenu à maintes reprises pendant les discussions sur le thème du piège, à la fois en France et au pays : "90\% sont piégés par cette situation. Voilà, on est piégés par cette situation parce que c'est une situation qui devient monotone... Alors on a - excusez-moi des mots - on devient esclaves, de nous-mêmes. Oui, parce qu'il faut travailler pour les petits-enfants aussi, et le petit-enfant, pour moi c'est un peu de trop, quoi" (Saleem, 60 ans, Guelmime, Maroc).

\section{Relations familiales et rôles genrés}

La discussion ci-dessus a mis en avant l'une des caractéristiques principales de ces résidents vieillissants de foyers de travailleurs migrants, à savoir la conservation au-delà de l'âge de la retraite du rôle social prédominant de breadwinner. À cet égard, leur expérience de la vieillesse diffère de la norme en vigueur dans les pays d'accueil européens. La littérature socio-gérontologique soutient que les personnes âgées, du moins dans les sociétés industrialisées contemporaines, sont confrontées à une "perte de rôle" à mesure qu'elles avancent en âge, et que le départ à la retraite est un tournant critique de ce processus ${ }^{20}$. Dans la mesure où ces travaux ont distingué les aspects genrés du vieillissement, il en ressort que les hommes âgés sont particulièrement visés par le sentiment de perte de rôle et de moyens ${ }^{21}$. Pour les hommes vivant dans les foyers de migrants, cette transition vers la séniorité est plus complexe et conditionnée en partie par les normes de la communauté d'origine. Contrairement à la situation en France, la vieillesse n'est pas synonyme de cessation d'activité économique ou sociale dans les pays maghrébins ou sahéliens, où l'institution sociale et juridique qu'est la retraite n'est pas largement ancrée ${ }^{22}$. En outre, en plus de conserver leur rôle de breadwinner, certaines des personnes interrogées, comme nous l'avons déjà noté, ont encore des enfants à lécole, un rôle très éloigné de ceux attendus pour cet âge en France. Les différences ethniques compliquent davantage le tableau. Ces complexités montrent que pour véritablement comprendre la situation des hommes en foyer de travailleurs migrants à lâge de la retraite, il est nécessaire d'entrecroiser de multiples dimensions de différenciation telles que le genre, lâge, la 
Carte orange de Layachi Ait-Baaziz datée du mois d'octobre 1980. Un document qu'il conserve précieusement, 30 mars 2015 @ Camille Millerand

génération et l'origine ethnique. Il apparaît notamment que les résidents nord-africains interrogés sont confrontés au risque de perdre des rôles qui leur seraient typiquement conférés à leur âge dans leur pays d'origine. L'obligation normative d'assumer le rôle genré de breadwinner peut prolonger l'absence et déclencher des conflits familiaux qui, à leur tour, sont susceptibles de remettre en cause ces valeurs patriarcales de la famille. Les femmes et les enfants laissés au pays gagnent en autonomie aux dépens des résidents retraités des foyers. Ces difficultés étaient moins présentes dans mes recherches menées auprès des résidents d'Afrique de l'Ouest. Nous comptons démontrer que ce contraste s'explique par des liens d'appartenance différents, distinguant la famille comme le point de référence dans le cas nord-africain et les liens plus larges centrés sur le village pour ce qui est de l'Afrique de l'Ouest.

\section{L'absence prolongée déstabilise le modèle familial patriarcal}

Au cours de mes conversations, les résidents nordafricains ont parfois exprimé une certaine rancœur envers l'autorité que les femmes ont pu acquérir. "Une femme c'est comme un portefeuille, a déclaré l'un d'eux, elle cache ton argent." Les femmes des résidents des foyers peuvent transformer le modèle familial du patriarcat en prenant le contrôle du budget du foyer, l'éducation des enfants et la discipline. Après une certaine période d'absence, beaucoup se plaignent d'avoir "perdu leurs repères" dans leur village d'origine et au sein même de leur famille. Par conséquent, perturbés par l'autonomie grandissante de leur famille, certains hommes ne restent que quelques 
mois chez eux. "On en a marre et puis on décide de revenir en France” (Kemal, 63 ans, Algérie).

Cette amertume et ces "rancunes" peuvent conduire au report du retour, même si les problèmes familiaux ne sont pas toujours ouvertement reconnus, comme l'illustre la citation suivante : "C'est comme je t'ai dit la dernière fois : les gens restent en France en raison de leur santé. Mais en fait c'est probablement un problème de stress avec leur famille. Ils supportent, ils supportent, et puis ils craquent, une maladie. Mais ils en parlent pas, ce sont des choses qui ne s'expriment pas.

Être absent du foyer pendant de longues périodes de sa vie professionnelle signifie pour un père qu'il ne peut pas voir ses enfants grandir, ni assister aux différentes étapes de leur développement.

perdre tes enfants" (Hamid, 70 ans, Agadir, Maroc). Être absent du foyer pendant de longues périodes de sa vie professionnelle signifie pour un père qu'il ne peut pas voir ses enfants grandir, ni assister aux différentes étapes de leur développement. Le père devient en quelque sorte un étranger pour ses enfants. Avec le temps, cet éloignement dans les faits peut conduire à un manque de respect des enfants envers leur père. Comme le fait remarquer Denis, un directeur de foyer : "Ils triment ici pour leurs enfants, mais leurs enfants ne sont pas reconnaissants." Lahcen, un représentant d'une ONG de développement qui travaille dans la région du Souss-Massa-Draâ au Maroc, attire l'attention sur l'une des "fautes majeures commises par les retraités... ils n'ont pas poussé leurs enfants à travailler. Le fils a 40 ans, mais il ne fait rien... il reste à la maison toute la journée et puis il demande de l'argent de poche pour aller prendre un café". Ce qui est encore plus dur pour certains pères est de découvrir que leurs enfants se comportent comme certains jeunes en France, comme des "délinquants". Kader (72 ans, Mostaghanem, Algérie) souligne la "déchéance morale" des jeunes dans son pays natal. Si ces histoires de normes familiales menacées caractérisent principalement les témoignages nord-africains, il est arrivé que les Africains de l'Ouest interrogés expriment leur inquiétude à ce sujet. Certains ont fait remarquer le matérialisme croissant des proches qui restent au pays, il se traduit par des demandes de biens matériels tels que des téléphones portables, des téléviseurs et autres gadgets de loisirs que les résidents sont censés rapporter en cadeaux à leur retour. Certains s'inquiètent d'un risque d'abus de la solidarité entre la famille proche et la famille étendue. Selon Moussa, la société de type traditionnel "est en train de se détruire, transformée en une société d'assistance et de dépendance... un confort parachuté" (Moussa, 64 ans, Mali).

Il pourrait être tentant d'interpréter ce discours sur les problèmes de la dépendance et de la délinquance comme les simples récriminations de maris à propos des exigences continuelles de leurs femmes et de l'ingratitude de leurs enfants. Cela s'est produit par moments au cours des entretiens menés avec des résidents retraités, mais un tel discours ne peut pas être réduit à de simples lamentations. L'émotion, qui s'intensifiait considérablement lorsque certains de ces hommes confiaient leur "esclavage", leur "exploitation", les "pressions", le "fardeau" et les "responsabilités", dépassait largement les chamailleries quotidiennes au sein des familles. La gravité des termes employés suffit à le prouver. Face à l'accumulation de ces conflits, le foyer a fini par devenir un "refuge". "Quand je reviens [en France], c'est vrai que je viens pour la santé, mais quand je suis là [au foyer], je suis soulagé un petit peu... C'est un refuge pour moi." (Saleem, 60 ans, Guelmime, Maroc).

\section{"Remote control" : I'exercice du pouvoir à distance}

Comme nous l'avons fait remarquer, le thème de l'absence et de la remise en cause des normes patriarcales revient beaucoup moins souvent dans 
les témoignages des résidents d'Afrique de l'Ouest. Cette partie s'appuie sur les témoignages recueillis durant nos travaux de terrain à Dembancané, dans la province de Matam au Sénégal ${ }^{23}$, de même que sur ceux menés en France. Les aînés expatriés originaires de Dembancané et d'autres villes en Afrique de l'Ouest que nous avons interrogés ont réussi à garder leur place au sein de leur communauté d'origine malgré leurs longues absences répétées. Les attentes normatives qui pèsent sur les personnes interrogées consistent à rendre service à la communauté d'origine une fois à la retraite, comme l'explique Waly (75 ans) du Mali : "Quand vous êtes retraité, vous devriez servir là-bas; vous retournez pour mieux servir votre village et votre famille." Pour ceux qui vivent une partie de l'année dans un foyer en France, ce type de services prend la forme de caisses de solidarité villageoises, qui jouent un rôle central pour les communautés sénégalaise, malienne et mauritanienne installées en France. Bien que l'origine des caisses villageoises remonte aux migrations internes antérieures vers Dakar et d'autres grandes villes, elles ont suivi les migrants en France après la Deuxième Guerre mondiale et ont souvent pour siège les foyers de travailleurs migrants ${ }^{24}$. En transplantant les modes de l'organisation sociale traditionnelle soninké en France, les structures locales de pouvoir fondées sur des critères hiérarchiques de genre et de génération sont reproduites et étendues à l'échelle transnationale ${ }^{25}$. Par le biais de ces caisses villageoises, les résidents des foyers de travailleurs migrants exercent leur pouvoir sur les populations locales en dépit de leur absence physique. Comme l'explique Catherine Quiminal, ces transferts de fonds ont "transformé l'absence de chacun en présence politique permanente ${ }^{26 "}$. Djimé mentionne sa forte implication dans la caisse de Dembancané et dans les projets de développement menés depuis ses premières années en tant qu'émigré : "Je peux rester pendant une heure à raconter des projets [de développement]! Ah, les projets, ça ne finit pas au village, parce que les immigrés, depuis notre époque, nous on cotise, on cotise sans arrêt, pour le développement de ce village. On a commencé par la poste, c'est nous-mêmes qui a construit notre poste. C'est nous-mêmes qui a construit notre école. C'est nous-mêmes qu'a... y en a plein, y en a plein. Le collège, on a financé; on a financé, avec les retraités aussi, les retraités, on est là, on a plus de 90 retraités ici. Chacun cotise pour le développement de ce village."

Comme l'indique le témoignage ci-dessus, les résidents continuent de cotiser même une fois à la retraite. La participation financière des migrants retraités reste attendue, même si elle est moindre que celle des migrants actifs en France. Les retraités à Dembancané sont censés participer à hauteur d'environ 50000 francs CFA par an (soit entre 75 et 80 euros, à l'époque où cette étude a été menée) ; les sommes varient selon les projets entrepris dans une année. Ce n'est pas une somme régulière, contrairement aux prélèvements mensuels ou trimestriels imposés aux villageois en âge de travailler vivant en France. Interrogeant un haut responsable de l'administration municipale de Dembancané sur la participation des retraités au développement du village, celui-ci nous a répondu : "Absolument. Politiquement, socialement, mais surtout économiquement... Ils ont été à l'origine de beaucoup de projets." Nous allons passer en revue chacun de ces domaines : politique, social et économique.

\section{La retraite ou l'âge de l'influence}

La contribution des retraités de Dembancané est avant tout politique, parce que ce sont souvent les aînés qui décident des projets de développement.

23. Dembancané est le village d'origine de près de 200 hommes résidant dans un des foyers de travailleurs migrants de la région parisienne qui a servi à ces travaux. Contrairement aux autres sites sur lesquels nous avons mené nos recherches en Afrique du Nord, nous avons délibérément désigné cette ville par son nom pour satisfaire la demande des aînés de "situer Dembancané sur la carte". Afin de protéger l'anonymat des témoignages, nous avons omis tout élément permettant l'identification, tels que l'âge, le nombre d'enfants ou le métier exercé en France. Comme pour toutes les autres personnes interrogées, un pseudonyme a été utilisé. 24. François Manchuelle, Willing Migrants. Soninke Labor Diasporas, 1848-1960, Athens, Ohio University Press, 1997. 25. Mahamet Timera, Les Soninké en France. D’une histoire à l'autre, op. cit. 26. Catherine Quiminal, "Retours contraints, retours construits des émigrés maliens", Hommes \& Migrations, n 1236, 2002, p. 40. 
L'inspiration pour les projets collectifs à Dembancané provient des vieux expatriés en France, ainsi que des aînés au village, qui forcément comptent un nombre important de migrants retraités rentrés au pays. Par exemple, le marché couvert est une initiative venue de France, alors que le nouveau collège et l'École 2 (une des deux écoles primaires) ont été initiés par les aînés du village. En effet, l'École 2 a été en quasi-totalité financée par les retraités revenus au pays, qui ont apporté 8 millions de francs CFA (plus de 12000 euros) sur
Du point de vue social la retraite en Afrique de l'Ouest pour les résidents des foyers est dépeinte comme une nouvelle jeunesse, à l'opposé de la société industrialisée contemporaine où la retraite serait perçue comme une "mort sociale". les 9 millions du budget total. Leur présence est également politique dans la mesure où les caisses villageoises servent à pallier l'absence d'intervention de l'État. Grâce à leurs émigrés, les villages parviennent à l'autosuffisance. En outre, du fait de la prolifération de ses services collectifs (eau, électricité, poste et télécommunications, un nouveau marché couvert, des écoles primaires et secondaires), Dembancané s'est mis à attirer les populations voisines, augmentant ainsi son prestige. En reconnaissance de cela, il s'est vu décerner le statut de "commune" en 2008 par le président de l'époque, Abdoulaye Wade, et a élu son premier maire en 2009. Ce n'est maintenant plus "un simple village", mais une véritable ville de 7000 habitants.

Du point de vue social, la retraite en Afrique de l'Ouest pour les résidents des foyers est dépeinte comme une nouvelle jeunesse, à l'opposé de la société industrialisée contemporaine où la retraite serait perçue comme une "mort sociale". Comme le souligne un haut responsable de l'administration municipale à Dembancané : “C'est à la retraite qu'ils mènent une vie active, socialement... Ils deviennent plus présents socialement." Samba, qui nous a hébergés durant notre séjour à Dembancané, est l'incarnation parfaite du dynamisme social des retraités là-bas. C'est indéniablement une figure importante du village : les gens viennent constamment lui demander conseil. Il est souvent sorti du matin au soir, occupé à s'entretenir avec les responsables politiques locaux ou à arbitrer des conflits entre des familles du village. Il n'est pas rare qu'à midi Samba montre déjà des signes de fatigue, suite aux nombreuses visites et démarches de la matinée.

En ce qui concerne la production économique, les efforts des anciens migrants revenus au pays ne se cantonnent pas aux affaires du village. Ils se portent également sur les cultures de millet, de riz et de sorgho qui entourent le village. Idrissa et Jaabé ont souligné le fait que ceux qui n'émigrent pas de Dembancané décèdent souvent à un jeune âge en raison du dur labeur que constitue le travail agricole. Toutefois, le rôle des anciens travailleurs migrants ne consiste généralement pas à servir de main-d'œuvre, il s'agit davantage pour eux de superviser et d'élaborer une stratégie, comme l'explique Amadou (64 ans, Goudiri, Sénégal) qui, lors de notre entretien en France, s'apprêtait à rentrer au pays pour superviser les semailles de la saison à venir. Le travail manuel à proprement parler pour les semailles relève davantage de la compétence des jeunes, les plus âgés dirigent les opérations et achètent le matériel. "Ici, c'est pas la même vie que les retraités d'Europe, parce qu'ici, quand tu rentres au village, donc c'est une nouvelle activité, une nouvelle condition. Parce qu'ici t'es tout le temps au travail, aller aux champs, l'agriculture, des démarches, la construction. On est là, on n'est pas au repos, en chômage! (Rires). En France, ils nous ont dit : 'Allez-y, allez-y au repos, reposez-vous : on vous paie.' Mais au contraire, on vient ici, on travaille !" (Djimé, Dembancané, Sénégal).

\section{Conclusion}

Regarder la situation des résidents de foyers de travailleurs migrants une fois à la retraite par le prisme de l'intersectionnalité nous éclaire sur la manière dont les relations au sein des familles transnationales et des communautés évoluent au fil du temps. Létude des motivations de cette (im)mobilité sur le 
tard sous un angle qui relie les différentes dimensions de la stratification sociale, à savoir le genre, l'âge et la génération, permet de révéler la complexité de ce que vivent ces hommes. L'intersection de ces dimensions avec l'origine ethnique, à travers une comparaison des témoignages des résidents nord et ouest-africains, a donné lieu à des résultats fort intéressants.

Dans le cas ouest-africain, les récits des résidents soninkés témoignent d'un attachement fort et durable au village d'origine qui puise ses racines dans les flux migratoires antérieurs vers les villes et les traditions d'entraide villageoise. Il ressort de ces entretiens qu'en dépit de longues absences du village, ces hommes ont pu entretenir une présence à plusieurs titres - politique, social et économique - grâce à leur contribution aux caisses villageoises, dont le siège est souvent au foyer de travailleurs migrants en France. Alors que l'ensemble des villageois bénéficient dans une certaine mesure de ces projets de développement, le fonctionnement de ces caisses villageoises signifie que ce sont des hommes (âgés) qui continuent à exercer le pouvoir le plus grand dans le village, perpétuant ainsi des hiérarchies basées sur le genre et la génération. À l'âge de la retraite, les Africains de l'Ouest peuvent ainsi s'appuyer sur le pouvoir politique, social et économique qu'ils ont été capables de maintenir, même de l'étranger, afin de faciliter leur réintégration dans leur village d'origine.

En revanche, les personnes nord-africaines n'ont pas accès à de tels mécanismes en raison d'un ensemble différent de valeurs et d'attentes dans leurs communautés d'origine, axé principalement sur la famille. L'analyse intersectionnelle des données a mis en évidence l'évolution au fil des ans du rôle des divers membres de la famille. Les témoignages nord-africains ont mis en avant la valeur quattachent les familles aux transferts de fonds des résidents de foyers de travailleurs migrants et à leur rôle genré en tant que breadwinner principal. Cependant, pour certains, un tel rôle ne peut être joué qu'à l'issue d'une séparation prolongée d'avec

la famille afin de pouvoir prétendre au minimum vieillesse. Ce qui conduit certains retraités à se retrouver "piégés" en France. Il a été constaté que cette prolongation du rôle de breadwinner par le travailleur migrant au-delà de lâge de la retraite, qui est une distorsion de la nouvelle économie de la migration de travail ${ }^{27}$, engendre des conflits au sein des familles non seulement entre les hommes et les femmes, mais aussi entre les générations. Cette absence prolongée a permis aux femmes et aux enfants d'acquérir l'autorité aux dépens des époux et pères absents. La valeur accordée par les familles aux transferts de fonds a ainsi contribué à réévaluer les structures familiales traditionnelles patriarcales. Dans certains cas, le foyer de travailleurs migrants est devenu un refuge, loin des problèmes familiaux.

Cette prépondérance donnée à la famille et au rôle de breadwinner plutôt qu'à la communauté villageoise au sens large et aux projets collectifs de développement fait que les résidents nord-africains interrogés ne peuvent pas tirer un prestige et un pouvoir semblables à ceux qui facilitent la réintégration de leurs homologues d'Afrique de l'Ouest. Cependant, vu la taille réduite de l'échantillon, nous nous garderons bien de généraliser. De

Pour les deux groupes de personnes interrogées, la réintégration à la vie de famille et à la vie communautaire est loin d'être acquise. même, il est important d'éviter toute exagération qui conduirait à dépeindre une situation toujours très facile pour les résidents d'Afrique de l'Ouest, et des scénarios forcément conflictuels pour ceux issus d'Afrique du Nord. Pour les deux groupes de personnes interrogées, la réintégration à la vie de famille et à la vie communautaire est loin d'être acquise. La décision de rentrer au pays, de circuler entre les deux ou de se réfugier dans un foyer de travailleurs migrants en France est motivée non seulement par les relations familiales, mais aussi par des questions de santé, des contraintes juridiques et administratives, ainsi que par les moyens de communication et de transports transnationaux ${ }^{28}$. 